\title{
A Case of Atypical Hemolytic Uremic Syndrome Successfully Treated with Eculizumab
}

\author{
$\begin{array}{lll}\text { B. Thajudeen } & \text { A. Sussman } & \text { E. Bracamonte } \\ & \text { B }\end{array}$ \\ Divisions of ${ }^{a}$ Nephrology and ${ }^{b}$ Pathology, University of Arizona, Tucson, Ariz., USA
}

\section{Key Words}

Hemodialysis treatment $\cdot$ Hemolytic uremic syndrome $\cdot$ Renal microcirculation $\cdot$ Renal insufficiency

\begin{abstract}
Atypical hemolytic uremic syndrome (aHUS) is a rare thrombotic microangiopathy (TMA) characterized by the triad of hemolytic anemia, thrombocytopenia, and acute renal failure. Eculizumab, a monoclonal complement $\mathrm{C} 5$ antibody which prevents the induction of the terminal complement cascade, has recently emerged as a therapeutic option for aHUS. We report a case of aHUS successfully treated with eculizumab. A 51-year-old male was admitted to the hospital following a mechanical fall. His past medical history was significant for rheumatic valve disease and mitral valve replacement; he was on warfarin for anticoagulation. A computed tomography scan of the head revealed a right-sided subdural hematoma due to coagulopathy resulting from a supratherapeutic international normalized ratio (INR). Following treatment with prothrombin complex concentrate to reverse the INR, urine output dropped and his serum creatinine subsequently increased to $247.52 \mu \mathrm{mol} / \mathrm{l}$ from the admission value of $70.72 \mu \mathrm{mol} / \mathrm{l}$. Laboratory evaluation was remarkable for hemolytic anemia, thrombocytopenia, elevated lactate dehydrogenase (LDH), low haptoglobin, and low complement C3. A renal biopsy was consistent with TMA, favoring a diagnosis of aHUS. Treatment with eculizumab was initiated which resulted in the stabilization of his hemoglobin, platelets, and LDH. Hemodialysis was terminated after 2.5 months due to improvement in urine output and solute clearance. The interaction between thrombin and complement pathway might be responsible for the pathogenesis of aHUS in this case. Eculizumab is an effective therapeutic agent in the treatment of aHUS. Early targeting of the complement system may modify disease progression and thus treat aHUS more effectively.
\end{abstract}


Thajudeen et al.: A Case of Atypical Hemolytic Uremic Syndrome Successfully Treated with Eculizumab

\section{Introduction}

Idiopathic atypical hemolytic uremic syndrome (aHUS) is a rare and severe thrombotic microangiopathy (TMA) characterized by the triad of hemolytic anemia, thrombocytopenia, and acute renal failure often caused by uncontrolled terminal complement activation affecting both children and adults [1]. Despite the use of supportive care, 33-40\% of patients die or have end-stage renal disease with the first clinical manifestation of aHUS, and $65 \%$ of all patients die, require dialysis, or have permanent renal damage within the first year after diagnosis despite plasma exchange or plasma infusion $[2,3]$. Terminal complement inhibition with eculizumab can safely reverse hematologic abnormalities and eliminate the need for dialysis [4]. Here, we report a case of idiopathic aHUS successfully treated with eculizumab.

\section{Case Report}

A 51-year-old Caucasian man was brought into the Emergency Department following a mechanical fall. His past medical history was significant for rheumatic heart disease, mechanical mitral valve replacement at the age of 29 years, repeat replacement at the age of 33 years, and left-sided stroke. He was on warfarin for anticoagulation (mechanical valve). His social history was significant for smoking (2-3 cigarettes/day) and occasional use of alcohol, while family history was significant only for diabetes mellitus.

On physical examination, his vitals were stable with a temperature $98^{\circ} \mathrm{F}$, blood pressure $113 / 79 \mathrm{~mm} \mathrm{Hg}$, pulse rate 88 beats/min, and respiratory rate 18 breaths/min. There was no rash except for mild ecchymosis over the right eye and helix of the right ear. His conjunctiva was pale but anicteric. There was mild pedal edema. A cardiac examination demonstrated a mechanical heart valve click, normal second heart sound, absent murmur, and no pericardial rub. His neurological exam revealed mild confusion and right-sided weakness. The rest of the examination was unremarkable.

A computed tomography of the head without contrast, performed in the Emergency Department, showed a right subdural hematoma. He was subsequently transferred to the Intensive Care Unit for observation and neurological monitoring. Laboratory tests showed a very high prothrombin time. Serum creatinine on the day of admission (day 1) was 70.72 $\mu \mathrm{mol} / \mathrm{l}$ (normal range: 53.04-97.24 $\mu \mathrm{mol} / \mathrm{l}$ ). He received 4-factor (II, VII, IX, and X) prothrombin complex concentrate for reversal of the high international normalized ratio in view of his subdural hematoma. By the next morning (day 2), he had become oliguric, and serum creatinine increased to $247.52 \mu \mathrm{mol} / \mathrm{l}$. Other laboratory results on day 2 are shown in table 1. Urine sediment showed granular casts. There were no dysmorphic red blood cells or cellular casts.

In view of the drop in hemoglobin and platelets, elevated lactate dehydrogenase (LDH), indirect hyperbilirubinemia, and acute kidney injury, the possibility of TMA secondary to hemolytic uremic syndrome/thrombotic thrombocytopenic purpura was considered. Complement studies showed C3 $0.70 \mathrm{~g} / \mathrm{l}$ (normal range: 0.82-1.85 g/l) and C4 0.19 g/l (normal range: $0.15-0.53 \mathrm{~g} / \mathrm{l}$ ). ANA, anti-dsDNA, hepatitis panel, HIV, anti-scl-70, ANCA and anticardiolipin antibody were all negative.

A peripheral smear demonstrated the presence of schistocytes consistent with microangiopathic hemolytic anemia. A renal ultrasound was unremarkable. A Tc-99m MAG3 scan demonstrated decreased perfusion to both kidneys and severely decreased cortical uptake. 
Thajudeen et al:: A Case of Atypical Hemolytic Uremic Syndrome Successfully Treated with Eculizumab

Renal Doppler study of the right kidney showed a resistive index of 0.69. Blood flow to the left kidney could not be assessed completely due to abdominal gas.

On day 4, a renal biopsy was performed which conformed TMA. The findings are outlined below (fig. 1, fig. 2, fig. 3).

A diagnosis of aHUS was made based on biopsy findings, serological data, and clinical history. A normal ADAMTS 13 and an equivocal antibody test to ADAMTS 13 ruled out thrombotic thrombocytopenic purpura. On day 3 , hemodialysis was initiated due to anuria and hyperkalemia. Plasma exchange with fresh frozen plasma was initiated on day 5 for treatment of aHUS $(60 \mathrm{ml} / \mathrm{kg}$ of fresh frozen plasma for restitution). Fresh frozen plasma was selected as the restitution fluid in view of the initial concern about genetic or inherited complement defects. Subsequent quantitative assays looking for genetic or inherited complement defects [factor B $239.7 \mu \mathrm{g} / \mathrm{ml}(127.6-278 \mu \mathrm{g} / \mathrm{ml})$, MCP CD46 positive, factor $\mathrm{H}$ $253 \mu \mathrm{g} / \mathrm{ml}(160-412 \mu \mathrm{g} / \mathrm{ml})$ and factor I $33.5 \mu \mathrm{g} / \mathrm{ml}(29.3-58.5 \mu \mathrm{g} / \mathrm{ml})]$ were within normal limits. Factor $\mathrm{H}$ antibody was negative. A genetic mutational analysis for the above described complement factors was unable to be performed due to denial from the patient's insurance company. Plasma exchange was discontinued after 2 days, and eculizumab was started after administration of a meningococcal vaccine (day 7). By day 8, the patient's platelets improved to $142 \times 10^{9} / \mathrm{l}$ and $\mathrm{LDH}$ decreased to $945 \mathrm{U} / \mathrm{l}$. Hemoglobin also stabilized. Eculizumab was continued at $900 \mathrm{mg}$ every week for a total of 5 weeks and then transitioned to 1,200 $\mathrm{mg}$ every 2 weeks. Urine output gradually improved and hemodialysis was terminated after 2.5 months. His estimated glomerular filtration rate at 6 months was $41 \mathrm{ml} / \mathrm{min} / 1.73 \mathrm{~m}^{2}$ as determined by the MDRD formula. The trends in LDH and platelets over time are represented in fig. 4. A computed tomography scan of the chest/abdomen/pelvis was also done to rule out infection or malignancy as precipitants for aHUS.

Informed consent was obtained from the patient for the publishing of this work.

\section{Discussion}

HUS is characterized by the presence of vascular abnormalities including glomerular endothelial injury and thrombosis [5]. It can be divided into 2 broad categories: typical (or otherwise D+HUS) and aHUS. D+HUS is more common in children and usually preceded by a history of diarrhea [5]. aHUS can be divided into idiopathic and secondary forms. The pathogenesis of idiopathic aHUS involves activation of the complement system [1]. Genetic aberration in 1 of the proteins of the alternative complement pathway can be found in at least $50 \%$ of idiopathic aHUS patients [6]. Genetic aberrations, however, are not the sole cause of complement activation in aHUS. An environmental factor, such as a complement trigger, is probably needed to develop the disease [5]. In more than half of the cases, the disease is precipitated by an infection, medication, or pregnancy [5]. Most cases with identifiable mutations are diagnosed during childhood; however, the disease may not manifest until adulthood [1].

The precipitation of renal failure following exposure to prothrombin complex concentrate is the most interesting aspect of this case. Prothrombin complex concentrate is a combination of blood clotting factors (including thrombin) used in the treatment of coagulopathy. Coagulation and complement pathways overlap in a spatiotemporal manner in response to common pathophysiologic stimuli, which results in unchecked activity of both systems [7]. It is already known that thrombin can directly promote activation of complement by cleavage of complement C5, thereby releasing $\mathrm{C} 5 \mathrm{a}$ and $\mathrm{C5b}$ [7]. The release of C5b is instrumental in the initiation of membrane attack complex, subsequent injury to the 
endothelium, and development of thrombosis [7, 8]. Coagulation factors have also been found to be associated with cleavage of complement factor C3. But whether this can explain the activation of an alternate complement pathway is not clear [8]. It is under this circumstance that the possible role of sporadic or inherited genetic aberration in the proteins of an alternate complement pathway was considered. Although quantitative assays for complement factors were normal in this case, it does not rule out an underlying genetic defect which can be confirmed only by a genetic assay. In this context, it is also notable that the pathogenetic mechanisms that induce and maintain the activation of the complement cascade are largely unknown [6].

Treatment options for aHUS include plasma exchange and complement factor 5 inhibitor (eculizumab). Persistence of hemolysis or lack of improvement in renal function after 35 daily plasma exchanges has been regarded as a criterion for uncontrolled TMA and is an indication for initiating eculizumab [5]. Eculizumab is a recombinant, humanized, monoclonal anti-C5 antibody which specifically binds to complement C5, thereby blocking the cleavage of $\mathrm{C} 5$ into $\mathrm{C} 5 \mathrm{~b}$ [1]. It is used in the treatment of patients with confirmed or suspected mutations in complement regulators and prevention of posttransplant recurrence of HUS [1]. Eculizumab is very expensive and treatment cost comes to 400,000 USD/year [1]. We were justified in the use of eculizumab in this case based on the general agreement that it is indicated if there is 1 of the following situations: a positive family history of HUS, recurrent HUS, or hypocomplementemia at presentation [1]. Moreover, it is also conceivable that eculizumab is more effective in the early treatment of aHUS to protect the kidneys from ongoing complement-mediated damage and to allow recovery from reversible changes [2].

There are not many reported cases in the literature in which eculizumab was used as first-line therapy of de novo aHUS in native kidneys. Ohanian et al. [9] reported the use of eculizumab as first-line therapy in a severe case of aHUS complicated by CNS involvement. Two prospective clinical trials have been conducted evaluating the safety and efficacy of eculizumab use in aHUS. These studies are not yet published, but their preliminary results, as reported by the Food and Drug Administration, are promising [10,11]. Patients in these studies were intolerant or resistant to plasma therapy. Two observations from these trials are relevant to our case regarding the timing of initiation of treatment and therapeutic response. In the first trial, earlier initiation of eculizumab was associated with a shorter duration of clinical manifestation of aHUS or shorter plasma exchange/plasma infusion, and resulted in greater improvement in the estimated glomerular filtration rate [11]. In the second study, 4 of the 5 patients who required dialysis at study entry responded and discontinued dialysis for the duration of eculizumab treatment [11]. The median duration of maintenance treatment in both trials was approximately 62-64 weeks [10,11]. At present, our patient will continue on eculizumab for at least 24 months, with evaluation for possible discontinuation at that time pending clinical status.

\section{Conclusion}

Terminal complement inhibition by eculizumab results in the termination of complement-mediated TMA, preventing further damage to renal parenchyma and subsequent improvement in renal function over time. This case report highlights the fact that early targeting of the complement system may modify disease progression and thus treat aHUS more effectively. It can be used not only during an acute episode but also for long-term prophylaxis, especially in those patients at high risk for recurrence. 


\begin{tabular}{l|l}
\hline \multicolumn{2}{l}{ Case Rep Nephrol Urol 2013;3:139-146 } \\
\hline DOI: 10.1159/000357520 & $\begin{array}{l}\text { ○ 2013 S. Karger AG, Basel } \\
\text { www.karger.com/cru }\end{array}$ \\
\hline
\end{tabular}

Thajudeen et al:: A Case of Atypical Hemolytic Uremic Syndrome Successfully Treated with Eculizumab

\title{
Disclosure Statement
}

\author{
All authors report no conflicts of interest.
}

\section{References}

1 Noris M, Remuzzi G: Atypical hemolytic-uremic syndrome. N Engl J Med 2009;361:1676-1687.

2 Caprioli J, Noris M, Brioschi S, Pianetti G, Castelletti F, Bettinaglio P, Mele C, Bresin E, Cassis L, Gamba S, Porrati F, Bucchioni S, Monteferrante G, Fang CJ, Liszewski MK, Kavanagh D, Atkinson JP, Remuzzi G: Genetics of HUS: the impact of MCP, CFH, and IF mutations on clinical presentation, response to treatment, and outcome. Blood 2006;108:1267-1279.

3 Noris M, Caprioli J, Bresin E, Mossali C, Pianetti G, Gamba S, Daina E, Fenili C, Castelletti F, Sorosina A, Piras R, Donadelli R, Maranta R, van der Meer I, Conway EM, Zipfel PF, Goodship TH, Remuzzi G: Relative role of genetic complement abnormalities in sporadic and familial aHUS and their impact on clinical phenotype. Clin J Am Soc Nephrol 2010;5:1844-1859.

4 Hodgkins KS, Bobrowski AE, Lane JC, Langman CB: Clinical grand rounds: atypical hemolytic uremic syndrome. Am J Nephrol 2012;35:394-400.

5 Loirat C, Frémeaux-Bacchi V: Atypical hemolytic uremic syndrome. Orphanet J Rare Dis 2011;6:60.

6 Mache CJ, Acham-Roschitz B, Frémeaux-Bacchi V, Kirschfink M, Zipfel PF, Roedl S, Vester U, Ring E: Complement inhibitor eculizumab in atypical hemolytic uremic syndrome. Clin J Am Soc Nephrol 2009;4:1312-1316.

7 Krisinger MJ, Goebeler V, Lu Z, Meixner SC, Myles T, Pryzdial EL, Conway EM: Thrombin generates previously unidentified C5 products that support the terminal complement activation pathway. Blood 2012;120:1717-1725.

8 Amara U, Rittirsch D, Flierl M, Bruckner U, Klos A, Gebhard F, Lambris JD, Huber-Lang M: Interaction between the coagulation and complement system. Adv Exp Med Biol 2008;632:71-79.

9 Ohanian M, Cable C, Halka K: Eculizumab safely reverses neurologic impairment and eliminates need for dialysis in severe atypical hemolytic uremic syndrome. Clin Pharmacol 2011;3:5-12.

10 Licht C, Muus P, Legendre C, Douglas K, Hourmant M, Delmas Y, Herthelius M, Delmas Y, Herthelius M, Trivelli A, Goodship T, Bedrosian CL, Loirat C: Long-term eculizumab therapy is effective in adolescent/adult patients with atypical hemolytic-uremic syndrome (aHUS) previously treated with chronic plasma exchange/infusion (PE/PI). Am Soc Nephrol, Kidney Week, Philadelphia, November 2011.

11 Licht C, Muus P, Legendre C, Douglas K, Hourmant M, Delmas Y, Herthelius B, Trivelli A, Goodship T, Bedrosian CL, Loirat C: Eculizumab is an effective long-term treatment in patients with atypical hemolyticuremic syndrome (aHUS) previously receiving chronic plasma exchange/infusion (PE/PI): extension study results. Am Soc Hematol, San Diego, December 2011. 
Case Reports in

Nephrology and

Urology

\begin{tabular}{l|l}
\hline Case Rep Nephrol Urol 2013;3:139-146 \\
\hline DOI: 10.1159/000357520 & $\begin{array}{l}\text { ○ 2013 S. Karger AG, Basel } \\
\text { www.karger.com/cru }\end{array}$ \\
\hline
\end{tabular}

Thajudeen et al.: A Case of Atypical Hemolytic Uremic Syndrome Successfully Treated with Eculizumab

Table 1. Laboratory results on day 2 of admission

\begin{tabular}{lrc}
\hline Laboratory results & Value & Reference range \\
\hline Hemoglobin, g/dl & 62 & $115-155$ \\
White blood cell count $\times 10^{9} / \mathrm{l}$ & 15.7 & $3.4-10.4$ \\
Platelets $\times 10^{9} / \mathrm{l}$ & 22 & $150-425$ \\
Haptoglobin, $\mathrm{g} / \mathrm{l}$ & $<0.8$ & $1.4-3.5$ \\
Fibrinogen, $\mu \mathrm{mol} / \mathrm{l}$ & 12.67 & $5.88-12.64$ \\
Prothrombin time (INR) & 2.3 & \\
Partial thromboplastin time, s & 32.1 & $29-35$ \\
Sodium, mmol/l & 131 & $136-145$ \\
Potassium, mmol/l & 6.1 & $3.5-5.1$ \\
Chloride, mmol/l & 96 & $101-111$ \\
Bicarbonate, mmol/l & 21 & $20-29$ \\
Urea nitrogen, mmol/l & 13.56 & $2.49-7.14$ \\
Phosphorus, mmol/l & 0.48 & $0.74-1.52$ \\
Creatinine kinase, U/l & 317 & $29-168$ \\
Total bilirubin, $\mu$ mol/l & 41.04 & $3.42-20.52$ \\
Direct bilirubin, $\mu$ mol/l & 6.84 & $0-8.55$ \\
Alanine transaminase, $\mathrm{U} / \mathrm{l}$ & 115 & $0-55$ \\
Aspartate transaminase, $\mathrm{U} / \mathrm{l}$ & 223 & $5-34$ \\
Alkaline phosphatase, $\mathrm{U} / \mathrm{l}$ & 79 & $125-243$ \\
Albumin, g/l & 34 & $34-48$ \\
Lactate dehydrogenase, U/l & 3,284 & $125-243$ \\
Lactic acid, mmol/l & 1.3 & $0.5-2.2$ \\
\hline & &
\end{tabular}

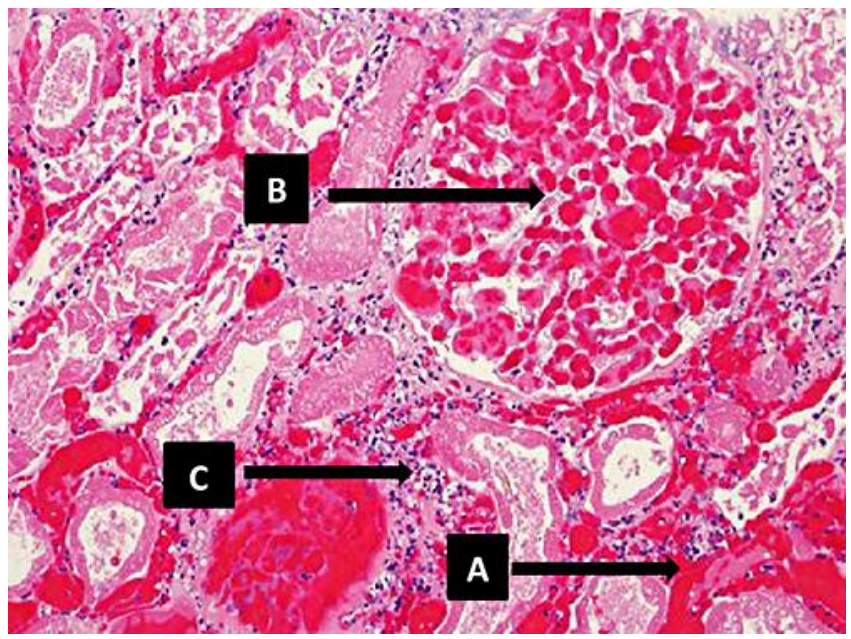

Fig. 1. The renal cortex demonstrates extensive coagulative necrosis and interstitial hemorrhage (A). H\&E. $\times 40$. Renal tubules are necrotic and there is marked glomerular congestion (B). A reactive acute inflammatory infiltrate is present within the interstitium (C). H\&E. $\times 200$. 


\section{Case Reports in \\ Nephrology and \\ Urology}

\begin{tabular}{l|l}
\hline Case Rep Nephrol Urol 2013;3:139-146 \\
\hline DOI: 10.1159/000357520 & $\begin{array}{l}\text { @ 2013 S. Karger AG, Basel } \\
\text { www.karger.com/cru }\end{array}$ \\
\hline
\end{tabular}

Thajudeen et al.: A Case of Atypical Hemolytic Uremic Syndrome Successfully Treated with Eculizumab

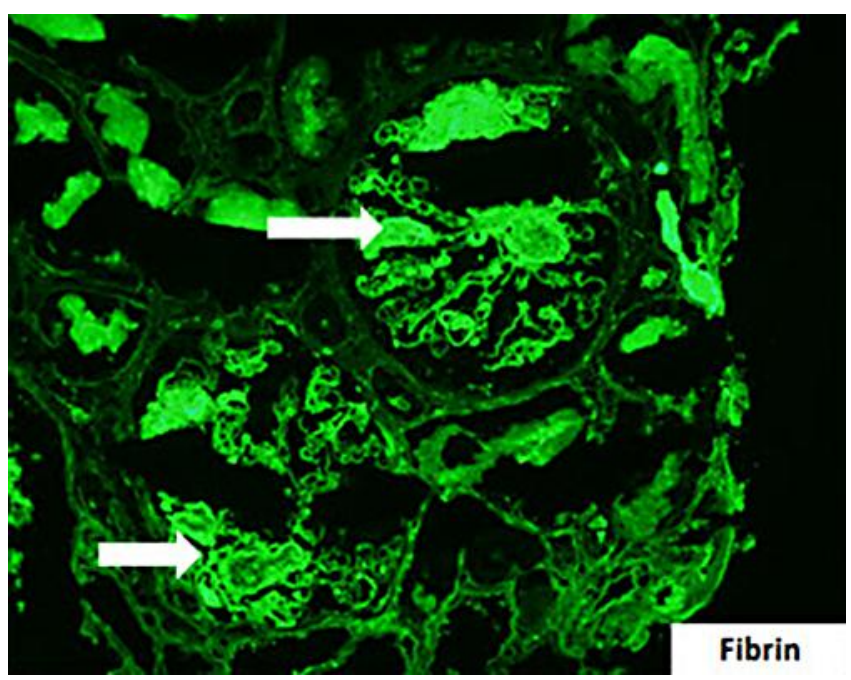

Fig. 2. Immunofluorescence shows segmental glomerular staining for fibrin $(3+)$, indicating intraglomerular thrombi (arrows). No specific immune complex deposition was identified. Anti-fibrin FITC. $\times 400$.

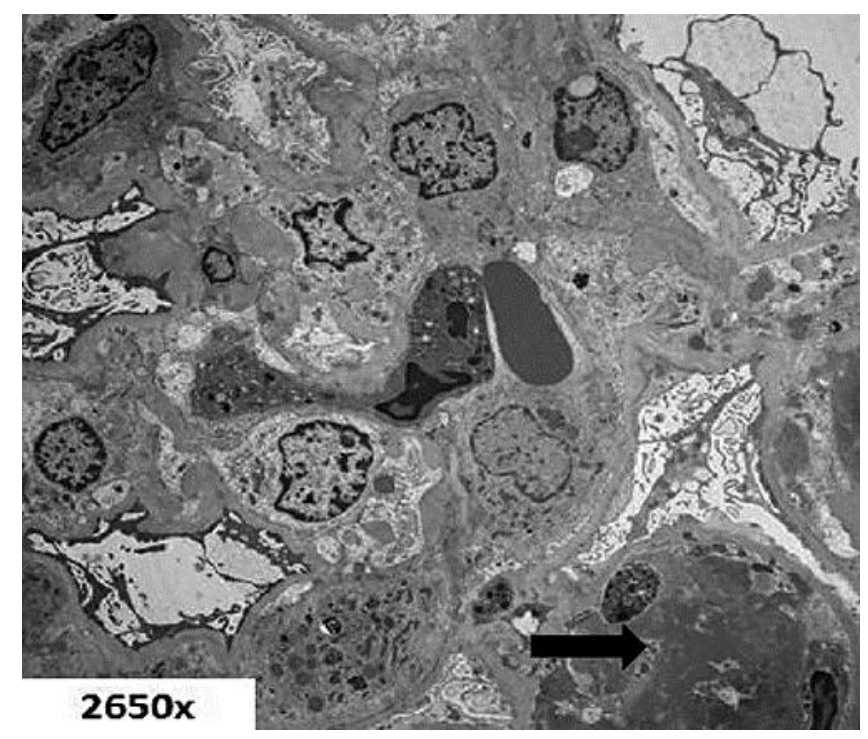

Fig. 3. Electron microscopy demonstrates capillary loops occluded by increased cells and occasional fibrin thrombi (arrow). There was mild swelling of the endothelial cell cytoplasm and patchy effacement of epithelial cell foot processes. No evidence of electron-dense deposits was seen $(\times 2,650)$. 
Thajudeen et al.: A Case of Atypical Hemolytic Uremic Syndrome Successfully Treated with Eculizumab

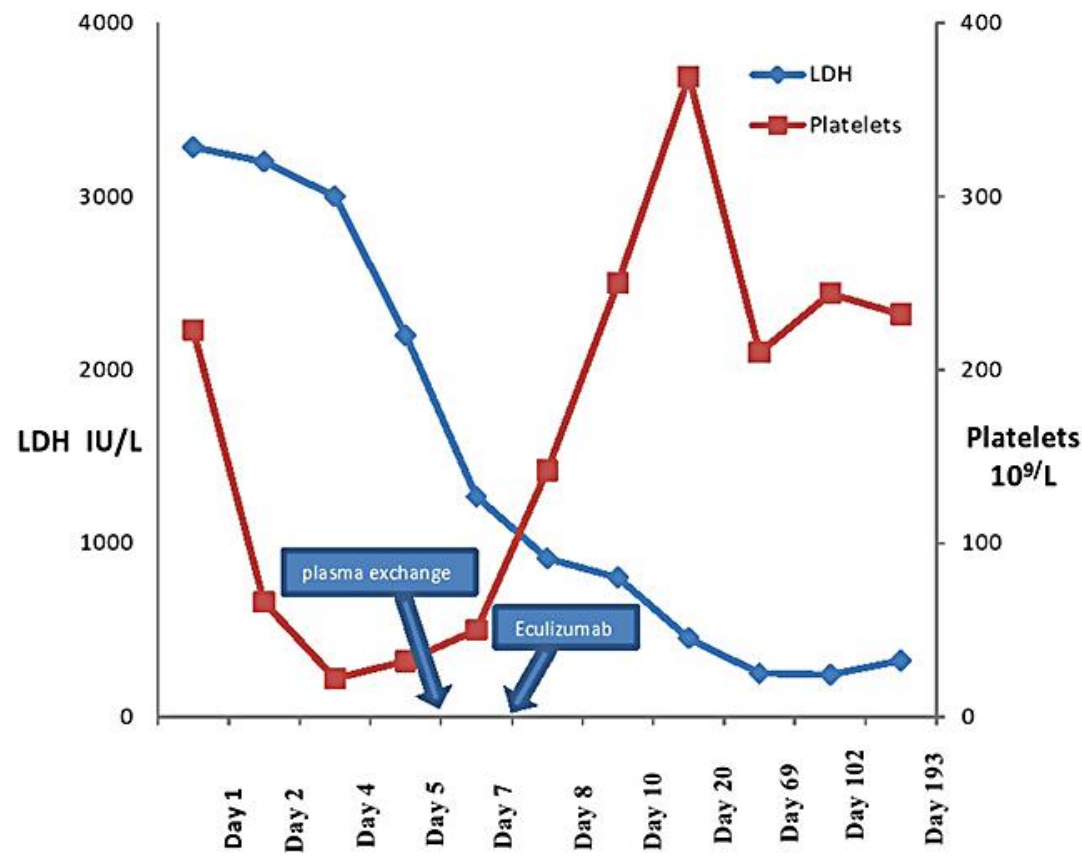

Fig. 4. Trends in LDH and platelets over time. 